Rabaska

Revue d'ethnologie de l'Amérique française

\title{
CRÉPA/Centre acadien (Université Sainte-Anne)
}

\section{Carmen d'Entremont}

Volume 14, 2016

URI : https://id.erudit.org/iderudit/1037491ar

DOI : https://doi.org/10.7202/1037491ar

Aller au sommaire du numéro

Éditeur(s)

Société québécoise d'ethnologie

ISSN

1703-7433 (imprimé)

1916-7350 (numérique)

Découvrir la revue

Citer ce document

d'Entremont, C. (2016). CRÉPA/Centre acadien (Université Sainte-Anne).

Rabaska, 14, 302-304. https://doi.org/10.7202/1037491ar d'utilisation que vous pouvez consulter en ligne.

https://apropos.erudit.org/fr/usagers/politique-dutilisation/ 


\section{Rapports des institutions}

\section{CRÉPA/Centre acadien}

Université Sainte-Anne

Pointe-de-l'Église

(Nouvelle-Écosse)

B0W $1 \mathrm{M} 0$

\section{ACADIE}

Téléphone : (902) 769-2114, poste 7204

Courriel : centre.acadien@usainteanne.ca

Toile : www.usainteanne.ca/centre-acadien
\end{abstract}

\section{Une année fructueuse et porteuse d'avenir}

Le Centre acadien a pour double mission de coordonner et de stimuler les activités de recherche dans le domaine des études acadiennes, et d'appuyer les chercheurs de l'Université Sainte-Anne dans les diverses étapes de leurs projets de recherche, tous domaines confondus. Afin de mieux assumer son rôle de Centre de recherche et d'études pluridisciplinaires acadiennes, le Centre acadien diffuse, depuis septembre 2015, une infolettre : Allez savoir. Celle-ci contribue à faire connaître les initiatives du Centre, à mettre en valeur les activités de recherche des membres de notre communauté universitaire et à communiquer les possibilités de recherche. Le Centre acadien organise de nombreuses activités en vue de renforcer l'excellence en recherche et en développement à l'Université Sainte-Anne, seul établissement postsecondaire de langue française en Nouvelle-Écosse. Nous visons ainsi à relier les activités de recherche dans notre institution au développement social, culturel et économique des communautés francophones de la Nouvelle-Écosse.

\section{Demandes de recherche et diffusion}

Environ une centaine de personnes de l'extérieur de l'université ont consulté notre centre au cours de l'année. En plus de recevoir des visiteurs du Canada (Québec, Ontario, Alberta, Saskatchewan et Colombie-Britannique) et des États-Unis (Massachussetts, Arizona, Maryland et Louisiane) intéressés par leur généalogie, nous avons également eu l'occasion d'orienter plusieurs chercheurs et amateurs universitaires. Parmi les sujets traités : les associations de la jeunesse catholique, la fondation de villages acadiens, la musique folklorique, la fête nationale des Acadiens, et l'industrie forestière de la Baie Sainte-Marie.

\section{Activités}

Cette année, le Centre acadien a organisé plus d'une douzaine d'activités. En voici un aperçu. 
- À l'automne, dans le cadre du Festival de la parole, le Centre a accueilli dans ses locaux un atelier de conte et d'écriture pour aînés. L'animation fut assurée par l'auteure acadienne, Georgette LeBlanc. Ensuite, Nicole Barrieau, conseillère principale à la recherche à la Faculté des études supérieures et de la recherche de l'Université de Moncton, présenta un atelier sur les stratégies de rédaction de demandes de subvention lors de la rencontre des employés de 2015. Près d'une vingtaine de professeurs et d'employés de l'Université Sainte-Anne en ont profité. Nous avons également reçu, à l'hiver, plus d'une vingtaine d'enseignants et d'enseignantes de l'École secondaire de Clare, venus sur le campus dans le cadre d'une journée pédagogique. L'objectif de leur visite était de prendre connaissance de nos ressources et d'envisager leur incorporation potentielle dans les salles de classe du Conseil scolaire acadien provincial (CSAP).

- De plus, quatre « 5 à 7 » de la recherche ont mis en valeur les chercheurs de notre institution. Parmi les conférenciers invités durant l'année, mentionnons Roger Gervais, professeur en sociologie, qui a mis en lumière l'exploitation d'un puissant logiciel (SPAD) pour analyser le contenu de milliers d'articles de journaux ; Andrea Burke-Saulnier, professeure en sciences de l'éducation, qui discuta des enjeux de la rétroaction corrective de la langue orale en salle de classe, en privilégiant les expériences des élèves ; et Caroline Fitzpatrick, professeure en psychologie, qui expliqua les disparités, au niveau de la santé et de la réussite, en traitant de l'importance du contrôle cognitif chez les enfants.

- Nous avons également organisé plusieurs lancements de livres afin d'appuyer et de promouvoir les publications des auteurs de la région et de la communauté universitaire. La célébration de la publication du roman Le diable est à la porte : entre ciel et terre de Gérald C. Boudreau, un de nos anciens directeurs, ainsi que celle des auteurs, Micheline Laliberté et Jean-Louis Robichaud, Vivre à la Baie : images d'un temps passé, entre autres, ont attiré des grandes foules.

- En avril, dans le cadre du Festival de Clare-té, le Centre acadien a reçu plus d'une trentaine de personnes à l'occasion de sa deuxième journée portes ouvertes. Nous avons lancé ce jour-là une exposition réunissant plus d'une cinquantaine de paniers divers, ayant tous un lien avec des gens de la région. De plus, une douzaine de participants ont eu la chance de suivre un atelier sur le tressage de paniers acadien et micmacs. Ce fut une expérience très enrichissante ! Au cours de l'activité, Carmen d'Entremont, qui termine actuellement un doctorat à l'Université de Moncton, anima une séance de contes autour du thème " paniers ». Plusieurs ont livré de précieux souvenirs personnels.

\section{Projets et expériences de travail}

Cette année, le Centre a pu offrir une expérience de travail à quatre étudiants de l'Université Sainte-Anne. Recruté par la Société acadienne de Clare, en collaboration avec le Centre, Adam Coulstring a mené une initiative intitulée « Les familles fondatrices de Clare ». Le but est de présenter de l'information historique qui soit ensuite accessible au public, notamment aux artistes, pour les appuyer dans la création de leurs œuvres. Mylène Comeau, qui a été embauchée comme assistante de recherche par l'entremise d'Emplois d'été Canada, a travaillé, entre autres, à la 
mise en ordre des collections et des ressources du Centre, ainsi qu'à l'organisation de l'exposition « 125 années d'histoire(s) à raconter ». À l'automne, dans le cadre d'un stage Coop, Nicole Thimot, étudiante en administration des affaires, a assumé un certain nombre de tâches importantes. En plus d'assurer l'accueil du Centre, elle a travaillé à l'importation de données de registres d'écoles, un projet commencé par Elaine LeBlanc. Nous espérons créer une base de données qui serait accessible depuis un site web. Enfin, au semestre d'hiver, nous avons accueilli comme stagiaire à temps plein Paul MacNeil, étudiant au programme d'éducation coopérative du département des sciences administratives. Il s'occupa de divers projets visant à rendre nos ressources plus accessibles, notamment la mise en ligne des descriptions de nos fonds d'archives, grâce aux initiatives de Cécile Pothier-Comeau, préposée aux archives.

CARMEn D'EnTREMONT

\section{Centre d'études acadiennes Anselme-Chiasson}

Université de Moncton

Moncton (Nouveau-Brunswick)

E1A 3E9
Téléphone : (506) 858-4085

Télécopieur : (506) 858-4530

Courriel : robert.richard@umoncton.ca Toile : www.umoncton.ca/umcm-CÉAAC

\section{Laboratoire de conservation et traitement}

Dans la campagne 2014-2015 du programme de subvention du Conseil des archives du Nouveau-Brunswick, deux projets d'un montant total de $3550 \$$ ont été attribués au secteur des archives de folklore et d'ethnologie du CÉACC. Conséquemment, Marc-André Haché s'est chargé du traitement audionumérique de 1300 bobines, spécialement par la validation du marquage informatique de 22000 enregistrements. Embauché à même deux subventions réparties sur 22 semaines et accordées par Jeunesse Canada au travail (Association pour l'avancement des sciences et des techniques de documentation) compagne 2015-2016, l'étudiant Bryan Poirier s'est permis d'identifier 500 morceaux de musique (surtout violon). À l'aide de sources documentaires (collections Athole et Skye, Ken Perlman), de musiciens et d'ethnomusicologues, il a complété et remplacé le titre de ces pièces conservées dans plusieurs collections des archives de folklore et d'ethnologie du CÉACC. Souvent, elles n'avaient subi initialement qu'une identification sommaire et typique, comme dans les exemples suivants : « reels à l'harmonica »; « vieilles jigs des îles »; « reel à bouche ». En 2015, Bryan Poirier a commencé la numérisation des quelque 17000 fiches de l'ancien fichier des archives de folklore du CéACC. Dans la majorité des cas, elles contiennent des informations biographiques qui n'ont pas été relevées ailleurs.

En 2015-2016, par le biais d'une contribution en espèce de l'Institut d'études acadiennes (IÉA), le CÉACC a procédé à la mise à niveau de quelques-uns de ses produits informatiques, spécifiquement son espace de conservation numérique (12 To), avec un nombre équivalent pour la sauvegarde, ainsi qu'à l'achat d'une nouvelle carte de son audionumérique externe (Roland Octa-Capture). Parallèlement, le CÉACC y 